\title{
TYPE AND COTYPE OF SOME BANACH SPACES
}

\section{MIECZYSLAW MASTYLO}

Institute of Mathematics

A. Mickiewicz University

Matejki 48/49

60-769 Poznań

Poland

(Received on June 11, 1990 and in revised form May 6, 1991)

\begin{abstract}
Type and cotype are computed for Banach spaces generated by some positive sublinear operators and Banach function spaces. Applications of the results yield that under certain assumptions Clarkson's inequalities hold in these spaces.
\end{abstract}

KEY WORDS AND PHRASES. Type, cotype, Clarkson's inequalities. 1991 AMS SUBJECT CLASSIFICATION CODE. Primary, 46B20.

\section{INTRODUCTION.}

Given a Banach space $X$, we let for any $n \in \mathbb{N}, 1 \leq p \leq 2 \leq q<\infty$ and $1 \leq s<\infty, K^{(p, s)}(X)$ and $K_{(q, s)}(X)$ be the smallest constants for which

$$
\begin{aligned}
K_{(q, s)}(X)^{-1}\left(\sum_{i=1}^{n}\left\|x_{i}\right\|^{q}\right)^{1 / q} & \leq\left(\int_{0}^{1}\left\|\sum_{i=1}^{n} r_{i}(t) x_{i}\right\|^{s} d s\right)^{1 / s} \\
& \leq K^{(p, s)}(X)\left(\sum_{i=1}^{n}\left\|x_{i}\right\|^{p}\right)^{1 / p}
\end{aligned}
$$

for every choice of $\left\{x_{i}\right\}_{i-1}^{n} \subset X$, where $\left\{r_{n}\right\}_{n-1}^{\infty}$ denotes the sequence of Rademacher functions defined by $r_{n}(t)=\operatorname{sign} \sin 2^{n} \pi t$ for $0 \leq t \leq 1$. If the left (resp. the right) inequality in $(1,1)$ holds, $X$ is of cotype $(q, s)$ (resp. type $(p, s)$ ). If $s=1$, we say that $X$ is of cotype $q$ (resp. type $p$ ) (see [6]).

The notions of type and cotype have appeared in various problems involving the analysis of vector valued functions or random variables. One of the great advantages of the classification of Banach spaces in terms of type and cotype is the existence of a rather satisfactory geometric characterization of these notions. For example Maurey and Pisier [8] showed that a Banach space $X$ is of type $p$ for some $p>1$ (resp. cotype $q$ for some $q<\infty$ ) iff $X$ does not contain $l_{1}^{n}$ 's (resp. $l_{\infty}^{n}$ 's) uniformly.

Note that if $X$ is of type $\left(p, p^{\prime}\right)$ with $K^{\left(p, p^{\prime}\right)}(X)=1,1<p \leq 2$ (resp. cotype $\left(p, p^{\prime}\right)$ with $K_{\left(p, p^{\prime}\right)}(X)=1$, $2 \leq p<\infty)$ and $1 / p+1 / p^{\prime}=1$, then $X$ verifies Clarkson's inequalities, i.e., for every $x, y \in X$, we have

$$
\begin{gathered}
\left(\frac{1}{2}\|x-y\|^{p^{\prime}}+\frac{1}{2}\|x+y\|^{p^{\prime}}\right)^{1 / p^{\prime}} \leq\left(\|x\|^{p}+\|y\|^{p^{1 / p}}\right. \\
\left(\operatorname{resp.}\left(\frac{1}{2}\|x-y\|^{p}+\frac{1}{2}\|x+y\|^{p^{1 / p}}\right)^{1 / p} \leq\left(\|x\|^{p^{\prime}}+\|y\|^{p^{\prime}}\right)^{1 / p^{\prime}}\right)
\end{gathered}
$$

Clearly (1.2), (1.3) implies that $X$ is uniformly convex. 
The well-known examples of Banach spaces for which the above inequalities hold are $L_{p}$-spaces (see [2]), $p$-Schatten ideals of compact operators on Hilbert spaces (see [9]), provided $1<p<\infty$.

In [10] Milman showed, using interpolation techniques that if $\Omega \subset R^{n}$ is a domain with minimally smooth boundary, then the inequality (1.2) applies to Sobolev spaces $W_{p}^{k}(\Omega)$ for $1<p \leq 2$. Further Cobos [3], using the above observation, proved that the inequalities (1.2) and (1.3) hold in $W_{p}^{k}(\Omega)$ for every domain $\Omega \subset R^{n}$ and $1<p<\infty$. In the same way Cobos and Edmunds in [4] showed that some Besov spaces and Triebel-Sobolev spaces verify Clarkson's inequalities.

In this paper we compute the type and cotype for spaces of large class of Banach spaces generated by some positive sublinear operators and Banach function spaces. This class includes for example: interpolation spaces determined by the real method of interpolation, Besov spaces, Triebel-Sobolev spaces (see [1],[11],[12]) $H^{p}$-spaces, an approximation space, $L^{p}(\mu, X)$-spaces and the other (see for example [5]). We also show that under some conditions Clarkson's inequalities hold in these spaces.

\section{PRELIMINARIES.}

Let $(\Omega, \mu)$ be a complete $\sigma$-finite measure space. If $X$ is a Banach space, we denote by $L^{\circ}(X)=L^{\circ}(\Omega, \mu, X)$ the $F$-space [i.e., complete and metrizable topological vector space of all equivalence classes of all $\mu$-Bochner measurable $X$-valued functions on $\Omega$. If $X=R$, then we write $L^{\bullet}-L^{\bullet}(\Omega, \mu)$.

A Banach space $E \subset L^{\circ}$ is called a Banach function space if $|x| \leq|y| \mu$-a.e. on $\Omega, x \in L^{\circ}$ and $y \in E$ imply that $x \in E$ and $\|x\|_{E} \leq\|y\|_{E}$.

Recall that a Banach function space $E$ is called $p$-convex (resp. p-concave), $1 \leq p<\infty$ if there exists a constant $M$ so that for all $x_{1}, \ldots, x_{n} \in E$, we have

$$
\begin{gathered}
\|\left.\left(\sum_{i=1}^{n}\left|x_{i}\right|^{p}\right)^{1 / p}\right|_{E} \leq M\left(\sum_{i=1}^{n}\left\|x_{i}\right\|_{E}^{p}\right)^{1 / p} \\
\left(\operatorname{resp} .\left(\sum_{i=1}^{n}\left\|x_{i}\right\|_{E}^{p}\right)^{1 / p} \leq M \|\left.\left(\sum_{i=1}^{n}\left|x_{i}\right|^{p}\right)^{1 / p}\right|_{E}\right) .
\end{gathered}
$$

The smallest possible value of $M$ is denoted by $M^{(p)}(E)\left(\right.$ resp. $\left.M_{(p)}(E)\right)$.

In what follows let $X$ be an $F$-space and let $S$ be a positive sublinear operator defined on $X$ taking values in $L^{\circ}=L^{\circ}(\Omega, \mu)$; that is for every $x, y \in X$ and any scalar $\lambda$ the following hold:

(i) $S x \geq 0$,

(ii) $S(\lambda x)=|\lambda| S x$,

(iii) $\quad s(x+y) \leq S x+S y$.

For a given Banach function space $E \subset L^{\circ}$ and injective operator $S: X \rightarrow L^{\circ}$, we define

$$
D_{E}(S)=\{x \in \mathcal{X}: S x \in E) \text {. }
$$

If $E=\left(L_{p},\|\cdot\|_{p}\right)$, we write in short $D_{p}$ instead of $D_{E}(S)$, where $\|x\|_{p}-\left(\int_{\Omega}|x|^{p} d \mu\right)^{1 / p}$ for $1 \leq p<\infty$.

Throughout the paper, we assume that $D_{E}(S)$ is a Banach space with the norm defined by

$$
\|x\|_{D_{E}(S)}=\|S x\|_{E} \text {. }
$$

We say that a pair $(E, S)$ is admissible provided that for any $A$ with $\mu(A)<\infty$, we have

$$
\chi_{A} S x_{n} \rightarrow 0 \text { in } E
$$

for every sequence $\left\{x_{n}\right\} \subset D_{E}(S)$ such that $x_{n} \rightarrow 0$ in $X$. Here $\chi_{A}$ is a characteristic function of $A$. 
TYPE AND COTYPE OF SOME BANACH SPACES

237

3. RESULTS.

Let $(T, v)$ and $(\Omega, \mu)$ be measure spaces. In the sequel for any $x_{1}, \ldots, x_{n} \in X$ and $f_{1}, \ldots, f_{n} \in L^{\circ}(T, v)$, we write

$$
\sum_{-1}^{n} f_{k} \otimes x_{k}(t)=\sum_{k=1}^{n} f_{k}(t) x_{k} \quad \text { for } \quad t \in T .
$$

An easy proof of the following lemma may be omitted.

LEMMA 3.1. Let $f_{1}, \ldots, f_{n} \in L^{\circ}(T, v)$. Then the following hold:

(i) For all $x_{1}, \ldots, x_{n} \in X$ and for any $\omega \in \Omega \quad S\left(\sum_{k=1}^{n} f_{k} \otimes x_{k}\right)(\omega) \in L^{\circ}(T, v)$.

(ii) For all $x_{1}, \ldots, x_{n} \in D_{E}(S),\left\|\sum_{k=1}^{n} f_{k} \otimes x_{k}\right\|_{D_{E}(S)} \in L^{\circ}(T, v)$.

(iii) If a measure space $(T, v)$ is finite, then for all $x_{1}, \ldots, x_{n} \in X$ and $f_{1}, \ldots, f_{n} \in L^{p}$,

$$
\left(\int_{h} S\left(\sum_{k=1}^{n} f_{k} \otimes x_{k}\right)^{p} d v\right)^{1 / p} \in L^{o}(\Omega, \mu) .
$$

THEOREM 3.1. Assume that $(T, v)$ is a finite measure space. Let $E$ be a Banach function space and let $f_{1}, \ldots, f_{n} \in L^{p}(T, v), 1 \leq p<\infty$.

(i) If $E$ is p-convex, then for all $x_{1}, \ldots, x_{n} \in X=D_{E}(S)$, we have

$$
\left\|\left(\int_{T} S\left(\sum_{k=1}^{n} f_{k} \otimes x_{k}\right)^{p} d v\right)^{1 / p}\right\|_{E} \leq M^{(p)}(E)\left(\int_{T}\left\|\sum_{k=1}^{n} f_{k} \otimes x_{k}\right\|_{X}^{p} d v\right)^{1 / p} .
$$

(ii) If $E$ is p-concave, then for all $x_{1}, \ldots, x_{n} \in X$, we have

$$
\left(\int_{T}\left\|\sum_{k=1}^{n} f_{k} \otimes x_{k}\right\|_{X}^{p} d v\right)^{1 / p} \leq M_{(p)}(E)\left\|\left(\int_{T} S\left(\sum_{k=1}^{n} f_{k} \otimes x_{k}\right)^{p} d v\right)^{1 / p}\right\|_{E} .
$$

PROOF. First of all, suppose that $f_{k}, k=1, \ldots, n$ are step functions. We can certainly assume that $f_{k}=\sum_{i=1}^{m} c_{i k} \chi_{A_{i}}$ with $A_{i} \subset T$ measurable, pairwise disjoint and $T=\bigcup_{i=1}^{m} A_{i}$. Then for $C=M^{(p)}(E)$, we have

$$
\begin{aligned}
& \left\|\left(\int_{T} S\left(\sum_{k=1}^{n} f_{k} \otimes x_{k}(t)\right)^{p} d v(t)\right)^{1 / p}\right\|_{E} \\
& =\left\|\left(\int_{T} S\left(\sum_{i=1}^{m}\left(\sum_{k=1}^{n} c_{i k} \chi_{A_{i}}(t) x_{k}\right)\right)^{p} d v(t)\right)^{1 / p}\right\|_{E} \\
& =\left\|\left(\int_{T}\left(\sum_{i=1}^{m} S\left(\sum_{k=1}^{n} c_{i k} x_{k}\right)^{p} \chi_{A_{i}}(t) d v(t)\right)\right)^{1 / p}\right\|_{E} \\
& =\left\|\left(\sum_{i=1}^{m}\left(v\left(A_{i}\right)^{1 / p} S\left(\sum_{k=1}^{n} c_{i k} x_{k}\right)\right)^{p}\right)^{1 / p}\right\| \|_{E}(\text { by } p-\operatorname{convexity)} \\
& \leq C\left(\sum_{i=1}^{m} v\left(A_{i}\right)\left\|S\left(\sum_{k=1}^{n} c_{i k} x_{k}\right)\right\|_{E}^{p}\right)^{1 / p}=C\left(\int_{T} \sum_{i=1}^{m}\left\|S\left(\sum_{k=1}^{n} c_{i k} x_{k}\right)\right\|_{E}^{p} \chi_{A_{i}}(t) d v(t)\right)^{1 / p} \\
& =C\left(\iint_{T}\left\|\sum_{k=1}^{n} f_{k} \otimes x_{k}(t)\right\|_{X}^{p} d v(t)\right)^{1 / p} .
\end{aligned}
$$


Now assume that in the sequence $\left\{f_{k}\right\}_{k=1}^{n}, f_{k}$ with $k=2, \ldots, n$ are step functions. Take any sequence $\left\{g_{j}\right\}_{,-1}^{\infty}$ in $L^{P}(T, v)$ of step functions such that $g_{j} \rightarrow f_{1} v$-a.e. on $T$, and $g_{j} \rightarrow f_{1}$ in $L^{P}(T, v)$. From this, we easily get that

$$
a_{j}=\left\|\left(\int_{T} S\left(g_{j} \otimes x_{1}+\sum_{k=2}^{n} f_{k} \otimes x_{k}\right)^{p} d v\right)^{1 / p}\right\|_{E} \rightarrow\left\|\left(\int_{T} S\left(\sum_{k=1}^{n} f_{k} \otimes x_{k}\right)^{p} d v\right)^{1 / p}\right\|_{E}
$$

and

$$
b_{1}=\left(\int_{T}\left\|g_{j} \otimes x_{1}+\sum_{k=2}^{n} f_{k} \otimes x_{k}\right\|_{X}^{p} d v\right)^{1 / p} \rightarrow\left(\int_{T}\left\|\sum_{k=1}^{n} f_{k} \otimes x_{k}\right\|_{X}^{p} d v\right)^{1 / p}
$$

Since $a_{j} \leq C b_{j}$ by previously proved inequality, we obtain desired inequality. Thus, by iterating the proof of (i) is complete. The proof of (ii) is similar.

Let us define on a $F$-space $X$ a family of semi-norm $|\cdot|_{\omega}$, by $|x|_{\omega}=S x(\omega)$ for every $\omega \in \Omega$. Now the main theorem of the paper is immediate.

THEOREM 3.2. Assume that $1<p<\infty$ and $1 \leq s<\infty$. Let $E$ be $a$ Banach function space and let $X=D_{E}(S)$.

(i) If $E$ is p-convex, $1<p \leq 2$, and s-concave, and for all $x_{1}, \ldots, x_{n} \in X$

$$
\left(\int_{0}^{1}\left|\sum_{k=1}^{n} r_{i}(t) x_{i}\right|_{\omega}^{s} d t\right)^{1 / s} \leq C_{1}\left(\sum_{i=1}^{n}\left|x_{i}\right|_{\omega}^{p}\right)^{1 / p} \quad \mu-a . e .,
$$

then $X$ is of type $(p, s)$ with $K^{(p, s)}(X) \leq C_{1}(p, s)=C_{1} M^{(p)}(E) M_{(s)}(E)$.

(ii) If $E$ is $p$-concave, $2 \leq p<\infty$, and s-convex, and for all $x_{1}, \ldots, x_{n} \in X$

$$
\left(\sum_{i=1}^{n}\left|x_{i}\right|_{\omega}^{p}\right)^{1 / p} \leq C_{2}\left(\int_{0}^{1}\left|\sum_{i=1}^{n} r_{i}(t) x_{i}\right|_{\omega}^{s} d t\right)^{1 / s} \quad \mu \text {-a.e. }
$$

then $X$ is of cotype $(p, s)$ with $K_{(p, s)} \leq C_{2}(p, s)=C_{2} M_{(p)}(E) M^{(s)}(E)$.

COROLLARY 3.1. If the conditions of Theorem 3.2 are satisfied with $s=p^{\prime}$ and $C_{1}(p, s)=1$ (resp. $\left.C_{2}(p, s)=1\right)$, then Clarkson's inequality (1.2) (resp. (1.3)) holds for $D_{E}^{\prime}(S)$.

PROPOSITION 3.1. Let $\left(L_{p}, S\right)$ be an admissible pair, $1 \leq p<\infty$. Assume that $D_{p} \subset \mathcal{X}$ with continuous inclusion and that $D_{p}$ is a non-closed subspace in $X$. Then $D_{p}$ is not type $r$ (resp. cotype $r$ ) for any $r>p($ resp. for any $r<p)$.

PROOF. The above assumptions imply that for any $\varepsilon>0, D_{p}$ contains $(1+\varepsilon)$-isomorphic copy of $l_{p}$ (see [7]). Since type and cotype is inherited by subspaces, then the proof is finished.

In the theory of type and cotype the type and cotype indices of a Banach space $B$ which are defined as follows

$$
\begin{aligned}
& p(B)=\sup \{p: B \text { is of type } p\} \\
& q(B)=\inf \{q: B \text { is of cotype } p\}
\end{aligned}
$$

are important (see [8] for details). 
COROLLARY 3.2. Assume that the assumptions of Proposition 3.1 are satisfied. Let $X=D_{p}$.

(i) If $1<p \leq 2$ and for all $x_{1}, \ldots, x_{n} \in X$

$$
\int_{0}^{1}\left|\sum_{i=1}^{n} r_{i}(t) x_{i}\right|_{\omega}^{p} d t \leq C \sum_{i=1}^{n}\left|x_{i}\right|_{\omega}^{p} \quad \mu-a . e .,
$$

then $p(X)=p$.

(ii) If $2 \leq p<\infty$ and for all $x_{1}, \ldots, x_{n} \in X$

$$
\left(\sum_{i=1}^{n}\left|x_{i}\right|_{\omega}^{p}\right)^{1 / p} \leq C \int_{0}^{1}\left|\sum_{i=1}^{n} r_{i}(t) x_{i}\right|_{\omega} d t \quad \mu-a . e .
$$

then $q(X)=p$.

PROOF. Since $L_{p}$ is $p$-convex and $p$-concave Banach function space, we have $X$ is of type $(p, p)$ for $1<p \leq 2$ (resp. cotype $(p, p)$ for $2 \leq p<\infty$ ) by Theorem 3.2. In order to finish the proof it suffices to apply a result of Kahane (see [6, Theorem 1.e.13]) and Proposition 3.1.

\section{EXAMPLES.}

We give two general examples injective and positive sublinear operators satisfying the inequalities given in Theorem 3.2.

Let $(\Omega, \mu)$ be a measure space and let $\mathcal{X}$ be an $F$-space. Fix $1<q<\infty$ and assume that $\left\{T_{k}\right\}_{k=1}^{\infty}$ is a sequence of injective linear operators, $T_{k}: X \rightarrow L^{\circ}(\Omega, \mu)$, such that

$$
S x(\omega)=\left(\sum_{k=1}^{\infty}\left|T_{k} x(\omega)\right|^{q}\right)^{1 / q}<\infty \quad \mu-a . e .
$$

Then obviously the operator $S: X \rightarrow L^{\circ}$ is injective, positive sublinear. For this operator, we have

PROPOSITION 4.1. (i) If $1<p \leq q \leq 2$, then for all $x_{1}, \ldots, x_{n} \in \mathcal{X}$

$$
\left(\int_{0}^{1}\left|\sum_{i=1}^{n} r_{i}(t) x_{i}\right|_{\omega}^{p^{\prime}} d t\right)^{1 / p^{\prime}} \leq\left(\sum_{i=1}^{n}\left|x_{i}\right|_{\omega}^{p}\right)^{1 / p} \quad \mu-a . e .
$$

(ii) If $2 \leq q \leq p<\infty$, then for all $x_{1}, \ldots, x_{n} \in X$

$$
\left(\sum_{i=1}^{n}\left|x_{i}\right|_{\omega}^{p}\right)^{1 / p} \leq\left(\int_{0}^{1}\left|\sum_{i=1}^{n} r_{i}(t) x_{i}\right|_{\omega}^{p^{\prime}} d t\right)^{1 / p^{\prime}} \quad \mu-\text { a.e. }
$$

PROOF. We have $K_{\left(p, p^{\prime}\right)}\left(l_{q}\right)=1$ for all $1<q \leq 2$ and by a duality argument $K^{\left(p, p^{\prime}\right)}\left(l_{1}\right)=1$ for all $2 \leq q<\infty$ (see [10]).

Now assume that $1<p \leq q \leq 2$. Then by $q^{\prime} \leq p^{\prime}$, it follows that

$$
\begin{aligned}
& \left(\int_{t}^{1}\left|\sum_{i=1}^{n} r_{i}(t) x_{i}\right|_{\omega}^{p^{\prime}} d t\right)^{1 / p^{\prime}}=\left(\int_{0}^{1}\left\|\sum_{i=1}^{n} r_{i}(t)\left\{T_{k} x_{i}(\omega)\right\}\right\|_{l_{q}}^{p^{\prime}} d t\right)^{1 / p^{\prime}} \\
& \leq\left(\int\left\|\sum_{i=1}^{n} r_{i}(t)\left\{T_{k} x_{i}(\omega)\right\}\right\|_{l_{q}}^{q^{\prime}} d t\right)^{1 / q^{\prime}} \leq\left(\sum_{i=1}^{n}\left\|\left\{T_{k} x_{i}(\omega)\right\}\right\|_{l_{q}}^{q}\right)^{1 / q} \\
& \leq\left(\sum_{i=1}^{n}\left\|\left\{T_{k} x_{i}(\omega)\right\}\right\|_{l_{q}}^{p}\right)^{1 / p}=\left(\sum_{i=1}^{n}\left|x_{i}\right|_{\omega}^{p}\right)^{1 / p}
\end{aligned}
$$


for all $x_{1}, \ldots, x_{n} \in X$. The proof of (ii) is similar.

Let $X$ be a Banach space and let $X=L^{\circ}(\Omega, \mu, X)$. Define an injective, positive sublinear operator $S$ : $X \rightarrow L^{\bullet}(\Omega, \mu)$ by

$$
S x(\omega)=\|x(\omega)\|_{x}, \quad \omega \in \Omega .
$$

Then the Banach space $D_{E}(S)$ is well-known and is denoted by $E(X)$. Clearly the inequality (3.1) (resp. (3.2)) is equivalent to the fact that $X$ is of type $(p, s)$ (resp. cotype $(p, s)$ ).

\section{REFERENCES}

1. LOFSTROM, J. and BERGH, J., Interpolation spaces (Springer-Verlag, Berlin-Heidelberg-New York, 1976).

2. CLARKSON, J. A., Uniformly convex spaces, Trans, Amer. Math. Soc. 40 (1936), 396-414.

3. COBOS, F., Clarkson's inequalities for Sobolev spaces, Math. Japonica 31 (1986), 17-22.

4. COBOS F. and EDMUNDS, D. E., Clarkson's inequalities, Besov spaces and Triebel-Sobolev spaces, Zeitschr. für Anal. 7 (3) (1988), 229-232.

5. COIFMAN, R. R., MEYER, Y. and STEIN, E. M., Some new function spaces and their applications to harmonic analysis, J. Funct. Anal. 62 (1985), 304-335.

6. LINDENSTRAUSS, J. and TZAFRIRI, L., Classical Banach spaces II: Function spaces (Springer-Verlag, Berlin-Heidelberg-New York, 1979).

7. MASTYLO, M., Banach spaces via sublinear operators, to appear.

8. MAUREY, B. and PISIER, G., Séries de variables aléatoires vectorielles indépendantes et propriétés géométriques des espaces de Banach, Studia Math. 58 (1976), 45-90.

9. McCARTHY, C. A., $c_{p}$, Israel J.Math 5 (1967), 249-271.

10. MILMAN, M., Complex interpolation and geometry of Banach spaces, Ann. Mat. Pura Appl. 136 (1984), 317-328.

11. TRIEBEL, H., Interpolation theory, function spaces, differential operators (VEB Deutscher Verlag der Wissenschaften, Berlin, 1978).

12. TRIEBEL, H., Theory of function spaces (Birkhäuser, 1983). 


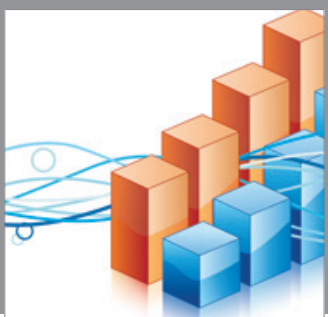

Advances in

Operations Research

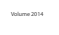

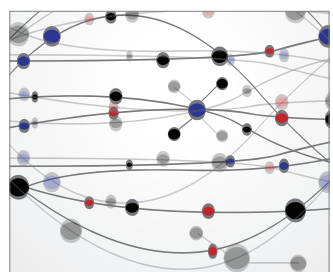

\section{The Scientific} World Journal
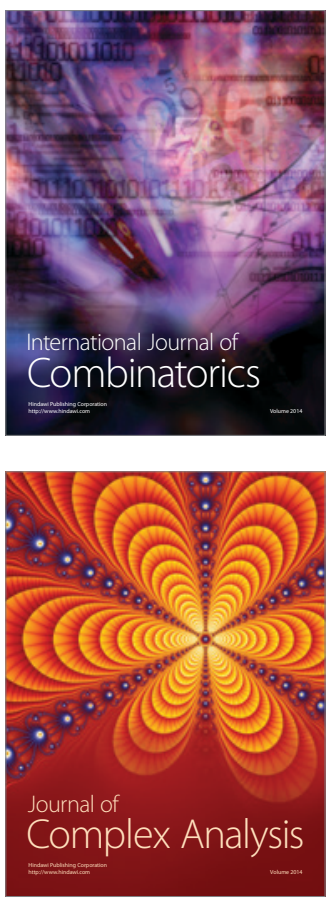

International Journal of

Mathematics and

Mathematical

Sciences
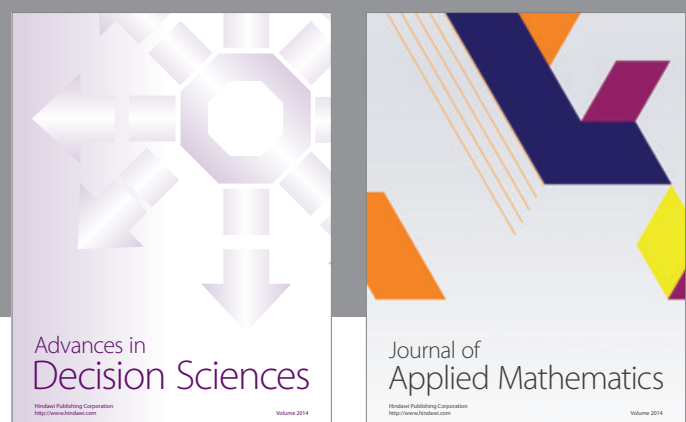

Journal of

Applied Mathematics
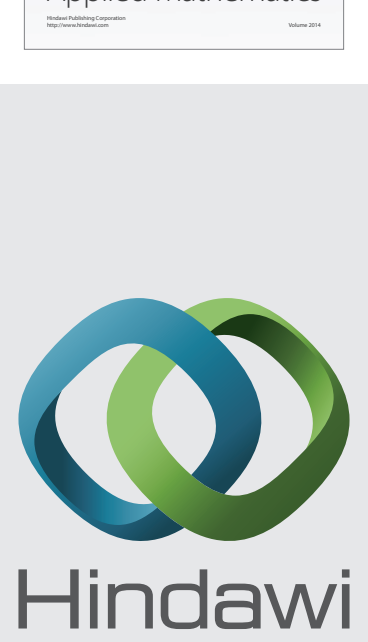

Submit your manuscripts at http://www.hindawi.com
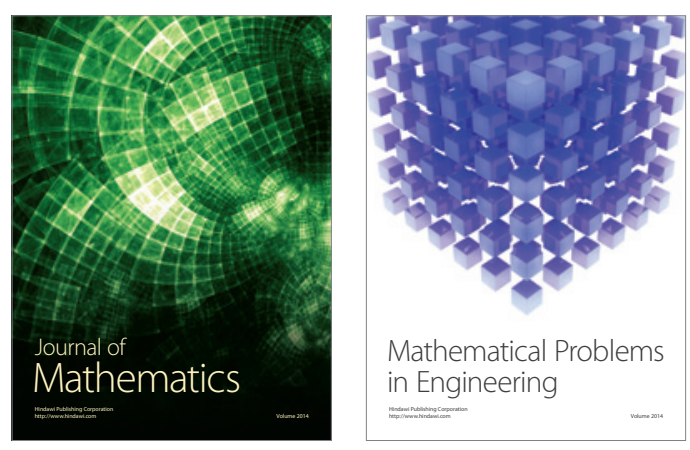

Mathematical Problems in Engineering
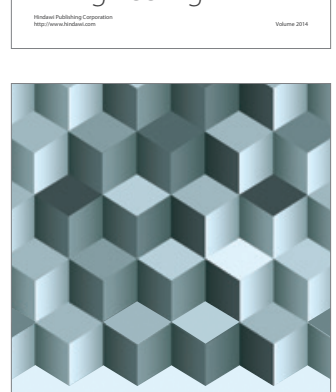

Journal of

Function Spaces
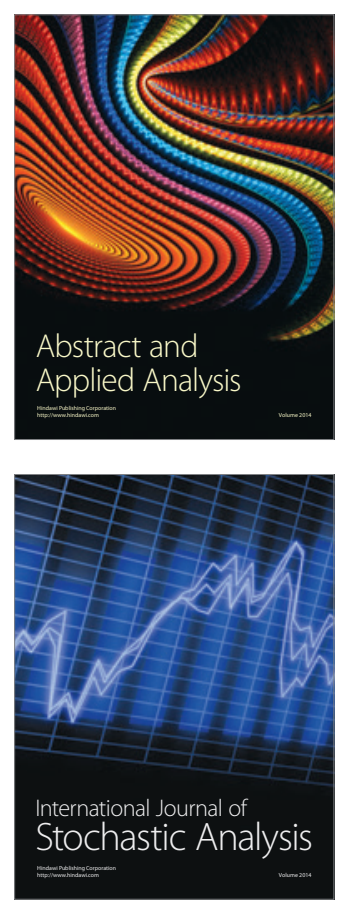

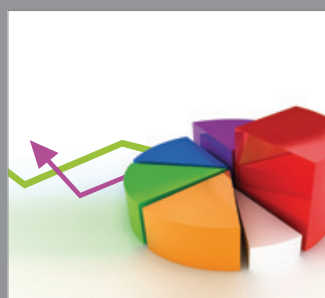

ournal of

Probability and Statistics

Promensencen
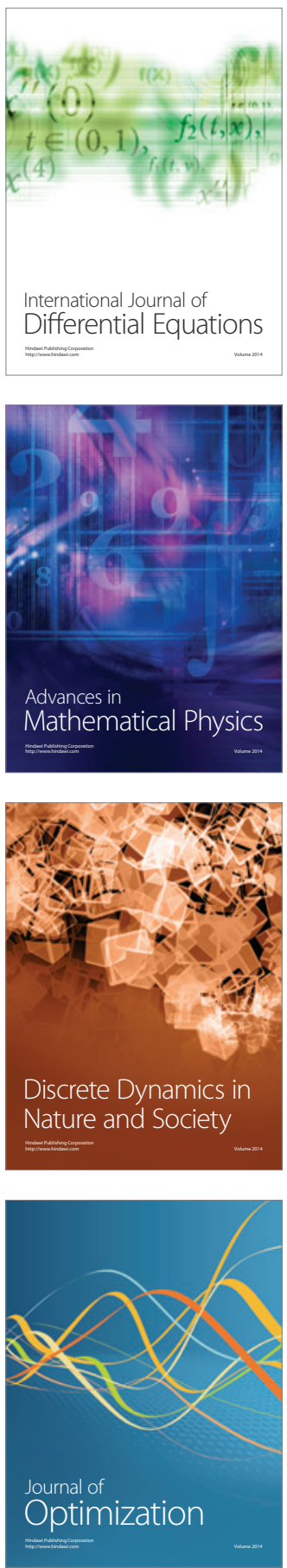